\title{
Auxiliando Professores de Engenharia de Requisitos a Utilizarem Jogos como Recursos Pedagógicos
}

\author{
Caio Steglich ${ }^{1}$ (Doutorando), Sabrina Marczak $^{1}$ (Orientadora)
}

\author{
${ }^{1}$ Programa de Pós Graduação em Ciência da Computação \\ Pontifícia Universidade Católica do Rio Grande do Sul (PUCRS) \\ Avenida Ipiranga, 6681 - CEP 90619-900 - Porto Alegre - RS - Brasil \\ Ingresso: 03/2019 - Qualificação: 03/2021 - Previsão Defesa: 03/2023 \\ caio.borges@edu.pucrs.br, sabrina.marczak@pucrs.br
}

Resumo. Diversos estudos na área de Educação em Engenharia de Software expressam as dificuldades ou desafios de aprendizagem de estudantes, ou como os estudantes reagem a uma nova abordagem de ensino utilizada pelo professor. Assim, poucos estudos exploram as dificuldades ou desafios enfrentados pelos professores de Engenharia de Software, especialmente quanto às tecnologias e materiais que eles poderiam utilizar para aumentar o interesse dos estudantes. Uma constante dificuldade dos professores de Engenharia de Requisitos é a preparação de aulas interativas, pois esta possui um conjunto extenso de conceitos raramente ilustrados em livros didáticos com exemplos práticos e concretos. Neste contexto, o objetivo deste trabalho é auxiliar os professores de Engenharia de Requisitos a identificarem jogos que se adéquem aos conteúdos que este professor irá ministrar em suas disciplinas sobre requisitos, de acordo com as suas necessidades e contexto de ensino. Para isto, será elaborado um ambiente inteligente, com conteúdo gerado pelos próprios usuários, ou seja, professores da comunidade, com jogos para apoiar práticas educacionais, onde os professores poderão avaliar cada jogo publicado no sistema bem como escrever sugestões e indicar o contexto de uso. Nesta tese, serão realizados diversos estudos, como Estudos de Caso, Revisões de Literatura e avaliação de protótipos, entre outros, para entender as demandas dos professores, propor um sistema de acordo com suas necessidades e avaliar sua eficiência no apoio aos professores. A maior contribuição esperada ao final desta pesquisa é um repositório inteligente sobre o uso de jogos nas disciplinas de Engenharia de Requisitos, que irá providenciar os jogos mais recomendados para o professor de forma inteligente.

Palavras-chave: Educação em Engenharia de Requisitos, Jogos em Engenharia de Requisitos, Sistema de Recomendação, Inteligência em Sistemas Educacionais, Estudo Empírico.

Evento CbSoft: SBES 


\section{Introdução e Caracterização do Problema}

Requisitos de Software são as descrições de quais funcionalidades o software deve possuir, os serviços que deve oferecer, e as restrições em seu funcionamento [1]. Estes requisitos devem refletir as necessidades dos usuários finais, servindo a algum propósito, como, por exemplo [1]. O estudo de requisitos como ciência é considerada Engenharia de Requisitos, sendo uma etapa importante do desenvolvimento de software, pois o que será desenvolvido irá de acordo com o que for especificado sobre os requisitos do sistema [1]. Contudo, a formação do profissional que irá lidar com estes requisitos é tema de discussão de diversos estudos, que exploram as mais diferentes abordagens e recursos de ensino, buscando ensinar aos estudantes os conceitos e técnicas mais adequadas para lidarem com esta disciplina da Engenharia de Software [2].

Um grande desafio dos professores no ensino de Engenharia de Requisitos (ER) é manter os estudantes motivados e com interesse em aprender sobre a área, pois a mesma demanda um estudo teórico aprofundado para a elaboração adequada dos requisitos geralmente acompanhado de livros didáticos ricos em conceitos mas carentes de exemplos práticos e concretos para auxiliar professor e estudantes no processo de ensinoaprendizagem. A grande multiplicidade de conceitos associada à variedade de técnicas e abordagens torna a identificação de materiais de qualidade ainda mais desafiante.

Uma abordagem promissora quanto ao ensino de requisitos é o uso de jogos como recursos pedagógicos, pois permitem aos estudantes aprenderem conceitos importantes enquanto se divertem, em um ambiente mais descontraído que o ambiente tradicional de ensino. Entretanto, da mesma forma, identificar jogos de qualidade que tratem um ou diversos conceitos de requisitos, e que se adéquem a um determinado contexto de ensino (por exemplo, desenvolvimento de atividades em grupo ou individual, alunos de graduação sem experiência prévia ou de pós-graduação com experiência de mercado) é também um grande desafio. Ainda, saber se a adoção deste jogo de fato contribuiu para o processo de ensino-aprendizagem e em qual contexto é algo distante de se ter à disposição.

Motivado por esta lacuna na literatura, este trabalho tem como objetivo auxiliar os professores de Engenharia de Requisitos a identificarem jogos para utilizarem no apoio ao processo de ensino-aprendizagem, através de um sistema computacional inteligente que possa recomendar um ou mais jogos para o ensino de um ou mais conceitos dentro de um determinado contexto de interesse. O sistema deve contar com um mecanismo de alimentação do catálogo de jogos pela própria comunidade de usuários bem como da avaliação da efetividade da recomendação no seu contexto de uso, servindo esta avaliação de base para recomendações futuras.

\section{Fundamentação Teórica}

\subsection{Ensino de Engenharia de Requisitos}

Existem diversos estudos sobre Ensino de Engenharia de Requisitos na literatura. Em 2015, Ouhbi e colegas [2] identificaram 79 estudos sobre o ensino de requisitos, concluindo que a maioria destes estudos apresentam uma proposta de metodologia de ensino ou relatam uma experiência na utilização de alguma abordagem ou recurso.

Experimentos realizados com estudantes para identificar a eficiência ou eficácia de uma abordagem ou recursos pedagógicos são constantes na literatura, como, por exemplo, 
o estudo de Berre e colegas [3], que buscaram entender o quão eficaz era a prática atual utilizada para que os estudantes aprendessem desenvolvimento de software orientado a modelos em uma disciplina de Engenharia de Requisitos. Outro relato de experiência é apresentado no estudo de Tiwari e colegas [4] que descrevem uma experiência sobre o uso de aprendizagem baseada em casos (do inglês: Case-Based Learning), com a intenção de melhorar o processo de aprendizagem em uma disciplina de Engenharia de Requisitos, onde os participantes eram 112 estudantes de pós graduação em um curso de Computação.

Hagel e colegas [5] estudaram como envolver clientes reais em projetos de software que seriam desenvolvidos em uma turma de Engenharia de Requisitos, e como isso pode motivar os estudantes e apoiar um entendimento mais profundo dos tópicos tratados na disciplina, aproximando os estudantes de situações reais que serão vivenciadas pelos futuros profissionais quando ingressarem na indústria. Tachikawa e colegas [6] explicam a respeito de uma experiência sobre o uso de uma combinação de treinamento sobre simular papéis, um sistema especialista no domínio do problema, como alternativa a um cliente e um sistema de agentes que desempenha o papel de consultor de melhores práticas.

\subsection{Uso de Jogos no Ensino de Engenharia de Requisitos}

O uso de jogos em áreas como Engenharia de Requisitos é explorado em diversos estudos, como, por exemplo, Souza e colegas [7] que realizaram um mapeamento da literatura sobre jogos utilizados no ensino de diversas subáreas de Engenharia de Software, sendo uma das exploradas a Engenharia de Requisitos. Entre suas contribuições, destaca-se que jogos podem ser tanto digitais, como ferramentas nas quais os estudantes simulam algum papel, quanto não digital, como jogos de tabuleiro ou de cartas voltados ao ensino.

Estudos sobre jogos no ensino de Engenharia de Requisitos apresentam desde relatos de experiência até revisões de literatura que investigam o uso destes jogos. Um exemplo de relato de experiência é o estudo de Garcia e colegas [8], que criaram um jogo digital chamado Biyubi, com o objetivo de auxiliar os estudantes a identificarem os stakeholders, utilizar técnicas de elicitação para entender as necessidades deste stakeholder e integrar, refinar e organizar estes requisitos. Os autores coletaram dados dos estudantes envolvidos no estudo, e perceberam que a utilização de jogos aumentou a motivação dos estudantes, bem como o entendimento sobre conceitos da área de Engenharia de Requisitos. Outro exemplo de relato de experiência é o estudo de Watson e Lipford [9] que investigaram o uso de um jogo sério (serious game), e a motivação de estudantes ao utilizarem um jogo sério para aprimorar o desenvolvimento de requisitos por parte dos estudantes. Estes autores apontam que 66,67\% dos estudantes (o estudo coletou dados de 185 estudantes) gostariam de ter mais jogos no ensino de outras disciplinas ou conteúdos, sendo que $42,8 \%$ destes realizaram mais atividades que as demandadas pela disciplina.

Existem também revisões de literatura sobre o uso de jogos na disciplina de Engenharia de Requisitos, como o estudo de Soo e Aris [10], que investigaram alguns dos jogos utilizados no tópico de ER de acordo com a literatura, suas classificações, objetivos de ensino e os resultados em suas implementações. Estes jogos investigados costumam ser implementados para plataformas web, na qual os estudantes podem simular algum papel, como analista de sistemas, gerente de projeto ou engenheiro de requisitos tendo, geralmente, como propósito ensinar aos estudantes a importância das especificações de um sistema ou de introduzir à prática de uma nova técnica aos estudantes. 
Por fim, alguns estudos propõem modelos sobre a criação de jogos educativos, como o estudo de Dalpiaz e Cooper [11] que elaboraram um framework que fornece sugestões de que pontos considerar quando deseja-se desenvolver um jogo para apoiar o ensino de Engenharia de Requisitos, sendo baseado nos seguintes pilares: Perfil do jogador (nível de conhecimento, papel, expectativas, etc), Motivação (ensino de um papel ou prática de uma técnica, etc), Conhecimento a ser desenvolvido (produtos, processos, etc), Como o jogo pode apoiar (simulação, gamificação, etc), Conteúdos a serem transmitidos (melhores práticas, padrões, etc), e Resultado esperado (aprendizado através do jogo, aumento da produtividade, etc).

Através da observação dos estudos identificados em revisões da literatura como as apresentadas por Soo e Aris [10] ou por Ouhbi e colegas [2], pode-se observar que poucos estudos demonstram a perspectiva do professor de requisitos sobre suas dificuldades ao prepararem suas aulas de forma mais interativa. Existem também frameworks sobre o que pensar quando irá se produzir um jogo para apoiar o ensino de requisitos [11], mas a literatura ainda não apresenta um framework ou guideline sobre como escolher um jogo para se utilizar no apoio ao processo de ensino-aprendizagem de requisitos.

\subsection{Sistemas de Recomendação no Ensino}

Sistemas de recomendação são aqueles que filtram dados através de conteúdo e perfis semelhantes, geralmente sendo utilizados em aplicações de e-commerce, mas também usada por redes sociais entre outras aplicações, visando ajustar o conteúdo as necessidades ou desejos de seus usuários finais [12]. Em áreas relacionadas a educação, a finalidade de um sistema de recomendação é oferecer apoio ao professor e às atividades de aprendizagem, apesar de poucos sistemas destes serem dedicados ao ensino [12].

Sommer e colegas [13] propuseram um sistema de recomendações com propriedades web para professores que os auxiliariam a encontrar quais plataformas de aprendizado digital seriam mais adequadas a suas demandas. Contudo, este estudo teve um cunho mais genérico quanto a educação de engenharias, apesar de suas recomendações e descrições dos algoritmos podem ser úteis na elaboração de outros sistemas de recomendação com outros propósitos. Não identificou-se, na literatura, um sistema de recomendações dedicado a auxiliar professores na escolha de jogos para o ensino de tópicos de ER.

\section{Metodologia de Pesquisa e Execução dos Estudos}

A proposta deste Doutorado é composta por 4 fases (Pré-Proposta, Caracterização do Problema, Proposta de Solução e Avaliação da Proposta) e 6 estudos principais (Survey, Revisão Sistemática de Literatura, Estudo de Caso com professores de ER, Entrevistas com Professores de ER, Avaliação com Especialistas e Múltiplos Estudos de Caso), sendo estes apresentados no Apêndice A. Esta mesma figura também apresenta os resultados parciais esperados e as etapas previstas para desenvolvimento do sistema.

\subsection{Pré-Proposta}

Inicialmente, realizou-se uma Survey [14] (Estudo 1) com professores de ER, com dados coletados através de um questionário online, que foi divulgado durante dois eventos relacionados ao tema em 2019, sendo esses o Workshop de Engenharia de Requisitos (WER) e o Simpósio Brasileiro de Engenharia de Software (SBES). 
Neste questionário, perguntou-se aos professores se possuem uma disciplina focada no tópico de ER, em quais cursos eles ministram estas disciplinas, quantos alunos costumam possuir em média nestas disciplinas, quais as abordagens ensinadas pelo pro-

fessor (exemplo: Ágil, Design Thinking, entre outras), se o professor utiliza jogos ou gamificação em suas disciplinas de ER e se estaria disponível para uma conversa futura em relação a esta pesquisa. Recebeu-se 31 respostas de professores de ER, e apenas 5 destes responderam utilizar jogos para o ensino de ER, 8 informaram utilizar alguma forma de gamificação e 18 informaram não utilizar nem jogos nem gamificação em suas disciplinas pelo desconhecimento ou dificuldade de selecionar material. Ainda, 15 professores mostrarem-se abertos para participar de estudos futuros e 13 explicam que poderão contribuir, mas gostariam de ser contatados antes para decidirem se irão participar.

Esta fase permite um breve entendimento do cenário do ensino de ER no Brasil, e seus resultados serão dispostos em um relatório técnico (em avaliação) submetido à Universidade na qual esta Tese de Doutorado está afiliada.

\subsection{Caracterização do Problema}

A fase de caracterização do problema conta com dois estudos, uma Revisão Sistemática da Literatura [15] (Estudo 2) e um Estudo de Caso com professores de ER [16] (Estudo 3). No Estudo 2, o objetivo foi identificar estudos que retratem o uso de jogos no ensino de ER, bem como os jogos disponíveis para uso. Neste momento, este estudo possui um protocolo formulado, com suas strings de busca e critérios de seleção de estudos baseados em um estudo anterior, realizado por Souza e colegas [7], que estudaram o uso de jogos no ensino de Engenharia de Software. No Estudo 2, ampliou-se a literatura identificada por [7] e selecionou-se apenas os estudos pertencentes a Engenharia de Requisitos (concluído). Através da extração de dados deste estudo vai-se identificar um catálogo de jogos(em andamento), que poderá servir de base para inspirar a definição das informações a comporem o registro de um jogo no sistema de recomendação. Os resultados devem ser publicados em um artigo científico (por exemplo, para a revista científica "Computers and Education”, ISSN: 0360-1315, ou “Computer Science Education”, ISSN: 1744-5175).

No Estudo 3, um Estudo de Caso com professores de ER será realizado para a caracterização de suas dificuldades e desafios enquanto ministram disciplinas de ER, bem como o que eles esperam de uma ferramenta que os apoiem na organização de atividades para o ensino de ER, ao prover recursos pedagógicos, como, por exemplo, um jogo. Neste momento, o protocolo de entrevistas está formulado, tendo sido revisado por dois pesquisadores experientes na área de Engenharia de Requisitos com mais de 10 anos de experiência. Os professores que serão convidados são os mesmos identificados no ensino de ER que não utilizam jogos no ensino requisitos (Estudo 1). Este estudo será submetido para alguma conferência renomada da área, como ICSE (International Conference in Software Engineering, em sua trilha de educação) ou SBES (Simpósio Brasileiro de Engenharia de Software, na sua trilha similar de educação).

\subsection{Proposta de Sistema}

Após a caracterização do problema, a proposta do sistema conta com dois estudos: um estudo baseado em entrevistas (Estudo 4) com professores de ER, para descobrir-se os requisitos fundamentais para o sistema que será proposto, e uma Avaliação com Especi- 
alistas [17] (Estudo 5), na qual os professores de ER poderão opinar sobre o protótipo e seus comentários serão utilizados para aprimorar-se a proposta do sistema.

Inicialmente, serão realizadas entrevistas com professores de ER (serão convidados os respondentes da survey (Estudo 1) que aceitaram participar de outros estudos) para identificar o que os mesmos esperam de um sistema como este. Após a captura destes requisitos, como parte do Estudo 5, o sistema será prototipado, e será iniciado um estudo cíclico de avaliação com especialistas (professores que utilizam jogos em suas disciplinas), para que estes avaliem as funcionalidades prototipadas [18] e opinem sobre como aprimorar o sistema como um todo, até que fique satisfatório na opinião destes professores. Ao final do Estudo 5, será possível a elaboração de um protótipo em alta fidelidade para a primeira versão do sistema, podendo partir para a etapa de desenvolvimento do sistema e gerando uma primeira versão do mesmo. Este estudo será submetido para publicação em alguma conferência, como o SBES (Simpósio Brasileiro de Engenharia de Software, em sua trilha de ferramentas).

Nesta etapa, ilustra-se o uso do sistema no seguinte cenário e forma: 1) Ao receber a alocação de uma disciplina na graduação pela primeira vez, o professor identifica no conteúdo programático tópicos sobre requisitos a serem ministrados; 2) Ao preparar as atividades para cada conteúdo programático e preparar suas estratégias pedagógicas, o professor procura por algum jogo que possa lhe auxiliar na introdução do conceito de requisito e suas classificações; 3) O professor se depara com a proposta do sistema de recomendações de jogos no apoio ao processo de ensino-aprendizagem de requisitos e indica seu contexto de uso-graduação, disciplina introdutória em ES, tópico conceitual sobre o que é um requisito de software e suas classificações, realiza a busca, e identifica 4 sugestões de jogos-3 virtuais, 1 de tabuleiro, estuda a descrição e avaliações de outros usuários destes jogos e escolhe pela opção do jogo virtual 1;4) O professor usa o jogo selecionado em aula; 5) Após a utilização, o professor retorna ao sistema, realiza sua avaliação do jogo utilizado e compartilha sua experiência com a comunidade. O sistema passa a usar essa nova avaliação como entrada para o algoritmo de recomendação.

\subsection{Avaliação do Sistema Proposto}

O Estudo 6 tem como propósito a realização de Múltiplos Estudos de Caso [16], no qual o sistema será utilizado por alguns professores de ER, para testar a primeira versão do sistema e coletar a opinião dos professores. Para isso, conta-se com um conjunto com 5 professores parceiros que já aceitaram participar desta etapa.

Através destes casos observados e da coleta da opinião dos professores de ER que utilizarão o sistema, será conduzido um ciclo de melhoria e otimização das funcionalidades do sistema [19], gerando assim uma versão estável do sistema e a possibilidade de mais um artigo para uma revista científica, explicando os resultados desta Tese de Doutorado como um todo.

\section{Comparação com Trabalhos Relacionados}

Os sistemas tutores inteligentes são recursos disponíveis que usam de inteligência para aprimorar a experiência do usuário, adaptando as suas dificuldades de aprendizagem. Como exemplo, Hilles e Naser [20] apresentam um sistema tutor inteligente para o ensino de banco de dados Mongo, apresentando a modelagem deste sistema para auxiliar 
os perfis de estudantes. A proposta desta Tese de Doutorado não será sobre um sistema tutor inteligente, uma vez que o objetivo do sistema proposto não é ensinar o professor, mas sim propor novos recursos (jogos) que se adéquem aos conteúdos que o professor irá ministrar em suas aulas sobre Engenharia de Requisitos.

A literatura também apresenta formas de avaliar um jogo quanto a sua utilidade, recursos, propósito, entre outras características. O modelo MEEGA, proposto por Petri e Wangenheim [21], possibilita a avaliação de jogos em diversas dimensões, como quanto a estética, aprendizagem, operabilidade, acessibilidade, interação social, diversão e satisfação. A proposta desta Tese não é fornecer um modelo de avaliação de jogos disponíveis para os professores, mas sim permitir que os mesmos avaliem a qualidade do jogo quando utilizados em aula e que esses professores compartilhem com a comunidade suas experiências. O modelo pode ser adaptado, por exemplo, para servir de base para a funcionalidade de avaliação dos jogos pelos professores, compondo o sistema.

Recentemente, Saule e colegas [22] apresentaram em um Webinar (um seminário na web) um projeto que os mesmos tem desenvolvido um repositório inteligente com conteúdos de Ciência da Computação. Este repositório tem os objetivos de classificar materiais, inspecionar os conteúdos cobertos em uma aula ou em um conjunto de materiais, estudar o alinhamento entre disciplinas de um mesmo currículo e entender como uma aula está sendo ministrada em diferentes universidades e programas. Apesar de jogos serem recursos que podem ser compartilhados em um repositório inteligente (como o CS materials destes autores), é necessário que essa inteligência auxilie o professor a identificar os jogos mais adequados, o que é o objetivo desta Tese, oferecer um sistema especializado em apoiar professores de ER a escolherem e utilizarem os jogos mais adequados ao conteúdo que será ministrado, não apenas provendo o recurso, mas permitindo que os professores avaliem os jogos e compartilhem suas experiências com a comunidade.

\section{Referências}

[1] I. Sommerville, Software Engineering. Addison-Wesley, 2011.

[2] S. Ouhbi, A. Idri, J. L. Fernández-Alemán, and A. Toval, "Requirements engineering education: A systematic mapping study," Requirements Engineering, vol. 20, no. 2, pp. 119-138, 2015.

[3] A. J. Berre, S. Huang, H. Murad, and H. Alibakhsh, "Teaching modelling for requirements engineering and model-driven software development courses," Computer Science Education, vol. 28, no. 1, pp. 42-64, 2018.

[4] S. Tiwari, D. Ameta, P. Singh, and A. Sureka, "Teaching requirements engineering concepts using case-based learning," in Proc. of the Int'l Workshop on Software Engineering Education for Millennials, (Gothenburg, Sweden), pp. 8-15, IEEE, 2018.

[5] G. Hagel, M. Müller-Amthor, D. Landes, and Y. Sedelmaier, "Involving customers in requirements engineering education: Mind the goals!," in Proc. of the European Conf. of Software Eng. Education, (Seeon, Germany), pp. 113-121, ACM, 2018.

[6] Y. Tachikawa and T. Nakamura, "Education for requirements elicitation using groupwork and role-play," in Proc. of the Global Engineering Education Conference, (Athens, Greece), pp. 780-783, IEEE, 2017.

[7] M. A. Souza, L. Veado, R. T. Moreira, E. Figueiredo, and H. Costa, "A systematic mapping study on game-related methods for software engineering education," Information and Software Technology, vol. 95, pp. 201-218, 2018. 
[8] I. Garcia, C. Pacheco, A. León, and J. A. Calvo-Manzano, "Experiences of using a game for improving learning in software requirements elicitation," Computer Applications in Engineering Education, vol. 27, no. 1, pp. 249-265, 2019.

[9] S. Watson and H. Richter Lipford, "Motivating students beyond course requirements with a serious game," in Proceedings of the Technical Symposium on Computer Science Education, (Minneapolis, USA), pp. 211-217, ACM, 2019.

[10] M. T. Soo and H. Aris, "Game-based learning in requirements engineering: An overview," in Proceedings of the IEEE Conference on e-Learning, e-Management and e-Services, (Langkawi, Malaysia), pp. 46-51, IEEE, 2018.

[11] F. Dalpiaz and K. M. Cooper, "Games for requirements engineers: Analysis and directions," IEEE Software, vol. 37, no. 1, pp. 50-59, 2018.

[12] A. C. Rivera, M. Tapia-Leon, and S. Lujan-Mora, "Recommendation systems in education: A systematic mapping study," in Proc. of the Int'l Conference on Information Theoretic Security, (Santa Elena, Ecquador), pp. 937-947, Springer, 2018.

[13] T. Sommer, U. Bach, A. Richert, and S. Jeschke, "A web-based recommendation system for engineering education e-learning solutions," in Engineering Education 4.0, pp. 293-302, Springer, 2016.

[14] B. Kitchenham and S. Pfleeger, "Principles of survey research: Part 1: Turning lemons into lemonade," ACM SIGSOFT Software Engineering Notes, vol. 26, no. 1, pp. 16-18, 2001.

[15] B. Kitchenham, O. P. Brereton, D. Budgen, M. Turner, J. Bailey, and S. Linkman, "Systematic literature reviews in software engineering-a systematic literature review," Information and Software Technology, vol. 51, no. 1, pp. 7-15, 2009.

[16] P. Runeson, M. Host, A. Rainer, and B. Regnell, Case Study Research in Software engineering: Guidelines and examples. John Wiley \& Sons, 2012.

[17] U. Flick, An Introduction to Qualitative Research. Sage Publications, 2014.

[18] L. Alperowitz, A. M. Weintraud, S. C. Kofler, and B. Bruegge, "Continuous prototyping," in Proceedings of the International Workshop on Rapid Continuous Software Engineering, (Buenos Aires, Argentina), pp. 36-42, IEEE, 2017.

[19] B. Fitzgerald and K.-J. Stol, "Continuous software engineering and beyond: Trends and challenges," in Proceedings of the International Workshop on Rapid Continuous Software Engineering, (Hyderabad, Índia), pp. 1-9, 2014.

[20] M. M. Hilles and S. S. A. Naser, "Knowledge-based intelligent tutoring system for teaching mongo database," European Academic Research, vol. 4, no. 10, pp. 87838794, 2017.

[21] G. Petri and C. G. von Wangenheim, "A method for the evaluation of the quality of games for computing education," in Proc. of the Workshops do Brazilian Conference of Informatics in Education, (Brasília, Brasil), pp. 951-960, br-ie.org, 2019.

[22] E. Saule, K. Subramanian, and J. Payton, "Cs materials: A system to classify cs courses against national curriculum guidelines," 2020. https://www.youtube.com/watch?v=fN4hcjU26g8. 


\section{A. O processo de metodológico proposto para Tese}

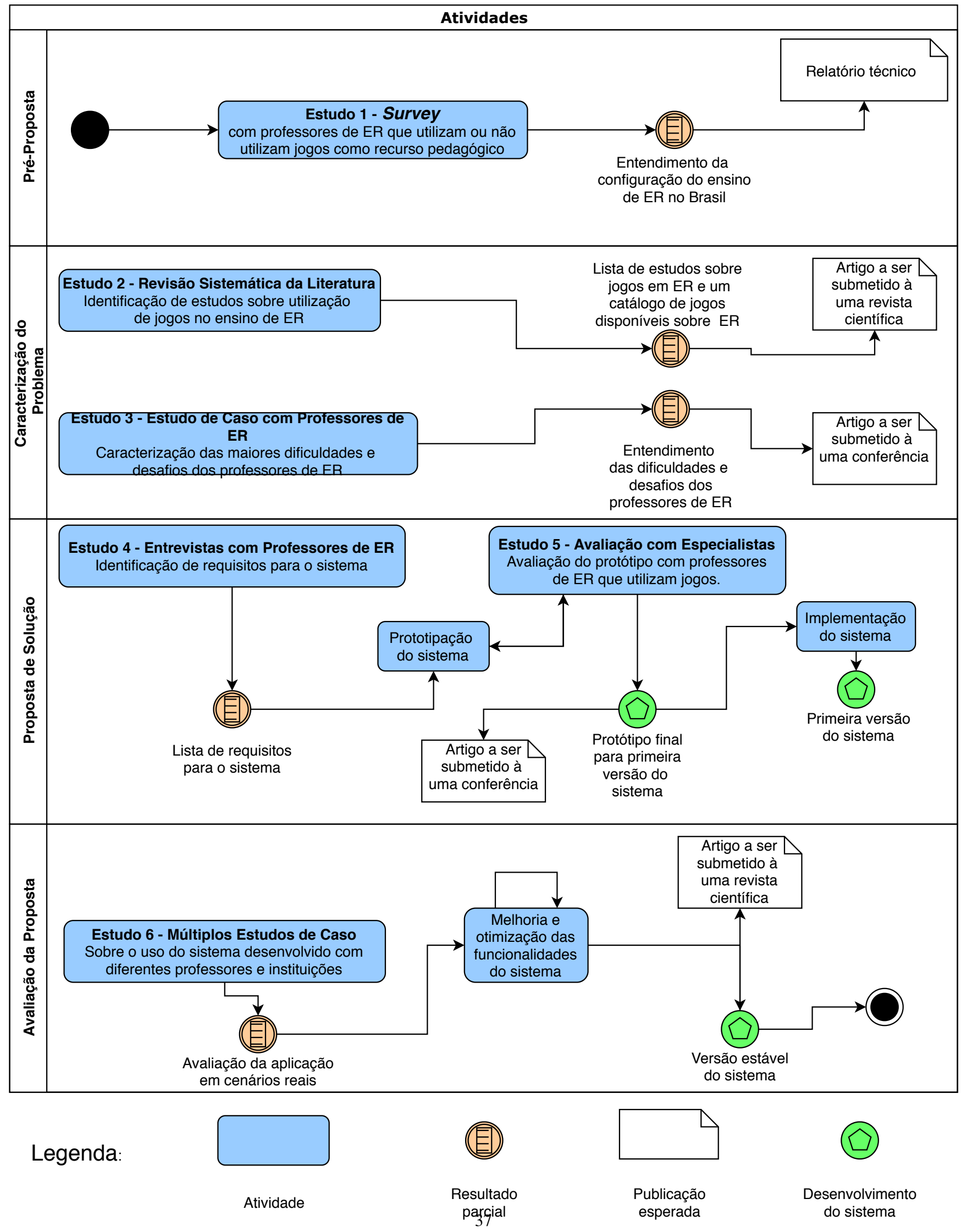

